\title{
Mapping of Incontinence Quality of Life (I-QOL) scores to Assessment of Quality of Life 8D (AQoL-8D) utilities in patients with idiopathic overactive bladder
}

\author{
Gang Chen ${ }^{1}$, Jonathan T Tan ${ }^{2 *}$ Kwong Ng${ }^{3}$, Angelo lezzi ${ }^{4}$ and Jeffrey Richardson ${ }^{4}$
}

\begin{abstract}
Background: The Incontinence Quality of Life (I-QOL) questionnaire is a commonly used and validated incontinence specific QOL instrument. The objective of this study is to develop an algorithm to map I-QOL to the Assessment of Quality of Life (AQoL) 8D utility instrument in patients with idiopathic overactive bladder (iOAB).

Methods: I-QOL and AQoL-8D scores were collected in a survey of 177 Australian adults with urinary incontinence due to $\mathrm{iOAB}$. Three statistical methods were used for estimation, namely ordinary least squares (OLS) regression, the robust MM-estimator, and the generalised linear models (GLM). Each included a range of explanatory variables. Model performance was assessed using key goodness-of-fit measures in the validation dataset.

Results: The I-QOL total score and AQoL-8D utility scores were positively correlated $(r=0.50, p<0.0001)$. Similarly, the three sub-scales of the $\mathrm{I-QOL}$ were correlated with the eight dimensions and two super-dimensions of the AQoL-8D. The GLM estimator, with I-QOL total score as the explanatory variable exhibited the best precision $(M A E=0.15$ and RMSE $=0.18$ ) with a mapping function given by AQoL-8D $=\exp (-1.28666+1.011072 *-Q O L / 100)$.

Conclusions: The mapping algorithm developed in this study allows the derivation of AQoL-8D utilities from I-QOL scores. The algorithm allows the calculation of preference-based QOL scores for use in cost-utility analyses to assess the impact of interventions in urinary incontinence.
\end{abstract}

Keywords: Urinary incontinence, Overactive bladder, HRQOL, I-QOL, AQoL, Mapping, Cross-walk, Utility

\section{Introduction}

Urinary incontinence (UI) is defined as any involuntary loss of urine. UI occurs at all ages, however, both prevalence and incidence of UI increase with age [1,2]. Incontinence is a significant health issue that has physical, social and economic implications on patients, as well as the broader community [3]. A study in the United States estimated the total cost associated with UI in the US as US\$19.5 billion [4]; an amount that was greater than the sum of the annual direct costs of breast, ovarian, cervical and uterine cancers [5].

\footnotetext{
* Correspondence: Tan_Jonathan@allergan.com

${ }^{2}$ Allergan Pty Ltd, Level 4, 810 Pacific Highway. Gordon, NSW 217 Melbourne, Australia

Full list of author information is available at the end of the article
}

Idiopathic overactive bladder syndrome (iOAB) is a common cause of urinary incontinence. As defined by the International Continence Society, $\mathrm{iOAB}$ is characterized by the presence of urinary urgency, often with UI, increased frequency ( $>8$ voids per day), and nocturia (ie, interruption of sleep $\geq 1$ time/night to urinate) [6]. Urinary incontinence due to $\mathrm{IOAB}$ is to be distinguished from other etiologies of incontinence such as neurogenic detrusor overactivity, where bladder dysfunction is due to a known neurological condition (eg, spinal cord injury), or stress incontinence, which is the involuntary loss of urine on physical exertion.

The ability to control one's elimination functions is a highly valued health outcome. Urinary incontinence and other $\mathrm{iOAB}$ symptoms have adverse impacts on multiple domains of health-related quality of life (HRQOL), 
affecting physical activity (eg, restrictions to daily activity due to leakage), sexual and interpersonal relationships, social interactions, and mental wellbeing [7]. The Australian Health Omnibus Survey found severe UI to be associated with a significant disutility of 0.32 based on the Assessment of Quality of Life (AQoL) and the EuroQOL five dimensions (EQ-5D) instruments [8]. Similarly, a national survey in the US, found that, after standardising for other personal characteristics, patients with UI had clinically and significantly poorer SF-36 scores across all domains compared to patients without UI [9].

The measurement and monitoring of HRQOL has become a key component for assessing the cost-effectiveness of medical interventions and health programs in clinical medicine research [10]. One common response to this need has been to include a disease specific instrument which has been specifically designed to measure dimensions of health which are important for the specific disease.

However, condition-specific measures do not provide 'utility' scores, which are necessary for the estimation of quality adjusted life years (QALYs) which are the unit of outcome in cost-utility analyses (CUA). CUA has become of increasing importance in the allocation of finite healthcare resources. For example, in Australia, Canada, and the United Kingdom, cost-per-QALY ratios are used by healthcare authorities in their appraisal of drug interventions. Consequently, a common approach to overcome this limitation is to develop mapping algorithms that transform condition-specific HRQOL scores into preference-based HRQOL utilities [11]. While generic utility instruments such as the SF-6D and EQ-5D can be utilised in any study regardless of the condition, this versatility comes at the expense of sensitivity to certain aspects of HRQOL that may be of importance to the conditions being studied. In the case of incontinence, a number of studies have shown that commonly used generic HRQOL instruments and multi-attribute utility instruments (MAUIs) are relatively insensitive to health dimensions that are affected by the treatment of UI [12-14]. In particular, these instruments fail to capture the full effect of poor sleep and psycho-social outcomes that are affected by incontinence. In the key study by McCallum [13], several MAUIs (ie, AQoL. EQ-5D, HUI3, SF-6D) were compared and the AQoL was identified as the instrument of choice for incontinence studies, as it provided the best coverage and sensitivity to HRQOL aspects impacted by incontinence. For this reason, the AQoL was selected for use in the present study in combination with the Incontinence Quality of Life Questionnaire (I-QOL). These two instruments are described in the Methods section.

The mapping algorithm will enable I-QOL scores observed in clinical trials for UI to be transformed into utility scores and facilitate the conduct of economic evaluations to inform decision decision-making with regards to healthcare resource allocation.

\section{Methods \\ Data}

A cross-sectional survey $(\mathrm{N}=1341)$ of individuals with iOAB in Australia, Canada, France, Germany, Italy, Spain, United Kingdom and the United States was conducted between December 2012 and March 2013 [15]. In only the Australian subgroup, both the I-QOL and AQoL instruments were administered. This data has been used in the present study to develop a mapping algorithm between the I-QOL and AQoL-8D.

Patients were recruited through the Quintiles (a contract clinical research organization) patient databases and registries. These registries are a voluntary medication monitoring service that allows interested individuals to be contacted to participate in medical surveys and studies relevant to their medical conditions. Respondents were screened to ensure that only subjects $\geq 18$ years of age, with previously diagnosed $\mathrm{iOAB}$, or those who had symptoms of $\mathrm{iOAB}$, were allowed to participate in the survey. Exclusion criteria included a predominance of stress UI, pregnancy, a history of neurological disorders, history of bladder disorders, including bladder stones and bladder outlet obstruction, a history of bladder or prostate cancer, and bladder reconstructive surgery.

Patient information was based on self-reported data through an online survey. Patients were granted access to the survey only after providing informed consent online. The study protocol was approved by the respective institutional review board, Ethical and Independent Review Services.

\section{Instruments}

\section{The incontinence quality of life questionnaire (I-QOL)}

The I-QOL, developed by Wagner [16] and Patrick [17], is a commonly used self-reported HRQOL instrument for people with UI. The I-QOL consists of 22 items, all of which use a five-point ordinal response scale in which 1 = extremely, 2 = quite a bit, $3=$ moderately, $4=$ a little, and $5=$ not at all.

The 22 items can be further grouped into 3 subscales: Avoidance and Limiting Behaviour (8 items), Psychosocial Impacts (9 items), and Social Embarrassment (5 items). The total I-QOL and 3 subscale scores are calculated by summing the unweighted item score and transforming them to a 100 point scale where $0=$ most severe, and $100=$ no problem . The instrument has been widely used and has been successfully validated for people with UI $[18,19]$. 
Assessment of quality of life eight dimension (AQoL-8D) Building upon the earlier AQoL MAUIs (ie, AQoL-4D and AQoL-6D), the AQoL-8D has significantly increased the content for health states pertaining to social and psychological problems [20], which are relevant in the study of incontinence. The AQoL-8D contains 35 items, these are grouped into eight dimensions (Independent Living, Relationships, Mental Health, Coping, Pain, Senses, Happiness and Self-Worth), which may be further grouped into two 'super-dimensions' (ie, physical and psychosocial/ mental). With four to six response levels for each of the 35 items, this allows for $2.37 \times 10^{23}$ possible health states. The time trade-off method was used for the preferencebased valuation of AQoL-8D health states. Utility scores were calculated from the 35 items using the AQoL-8D scoring algorithm (Version 13), available at www.AQoL. com.au. Although AQoL-8D has not been widely used to date, it has been successfully tested for reliability and validity [21]. Recent evidence indicates that AQoL-8D has greater sensitivity to the psycho-social dimensions of QOL (which are relevant in the study of incontinence) than the other utility instruments in common use [22].

\section{Statistical analysis}

The survey data were used to estimate a mapping algorithm between the I-QOL and AQoL-8D that can be applied for other studies. The approach is a widely used strategy in mapping analysis and is referred to in the literature as the 'transfer to utility regression technique' [23].In this study we adopted two model specifications. The first used I-QOL total scores and the second used the I-QOL subscale scores as the independent variable (s). Two demographic variables (age and gender) were included in the models which were specified as the following equations:

- Model 1: I-QOL total score model

$$
A Q o L 8 D=\alpha_{0}+\beta \cdot I Q O L+\alpha_{1} \cdot A G E+\alpha_{2} \cdot G E N D E R+\mu
$$

- Model 2: I-QOL subscale score model

$$
\begin{aligned}
A Q o L 8 D= & \alpha_{0}+\gamma_{1} \cdot I Q O L_{P S}+\gamma_{2} \cdot I Q O L_{S E}+\gamma_{3} \\
& \cdot I Q O L_{A L B}+\alpha_{1} \cdot A G E+\alpha_{2} \cdot G E N D E R+\mu
\end{aligned}
$$

where $A Q o L 8 D$ is the AQoL-8D utility; IQOL is the I-QOL total score; $I Q O L_{P S}, I Q O L_{S E}$, and $I Q O L_{A L B}$ are the scores of Psychosocial Impacts scale, Social Embarrassment scale, and Avoidance and Limiting Behaviour scale respectively; $A G E$ is a continuous variable for the respondents' age; GENDER is a dummy variable that equals 1 if the respondent is male and 0 otherwise; $\alpha_{0}$ is a constant, $\alpha_{1}, \alpha_{2}, \beta$, and $\gamma$ are the coefficients to be estimated, $\mu$ is the error term. The squared terms of the I-QOL (total/scale) scores were also considered in the relevant models. In the final models, only the explanatory variables which were statistically significant $(\mathrm{p} \leq 0.05)$ were retained. A stepwise regression technique with forward selection [24] was used to choose the "best" combination of predictors.

Three statistical techniques were adopted in the study to estimate the two models.

1. Firstly, an ordinary least squares (OLS) estimator was used. This has been the most widely used technique in the literature [11,23].

2. Secondly, an effective robust estimator, the MM estimator [25] was used. This has recently been proposed for mapping analysis by Chen [26]. The justification for using robust estimators lies in the fact that the OLS estimator is highly sensitive to sample outliers.

3. Lastly, the generalised linear model (GLM) was used, which allows for the non-normal distribution of dependent variables (e.g. left/negatively skewed utility scores) [27]. For the GLM estimation a choice must be made between the type of 'family estimate' (e.g. Gaussian, inverse Gaussian, binomial, gamma) and the link function (e.g. identity, log, logit, cloglog, log-log, log-complement, power).

Based on the goodness-of-fit results in the validation analysis, the Gaussian family with $\log$ link was chosen as the most appropriate method.

Other popular estimators that are widely adopted in the mapping analysis also include the Tobit estimator, the censored least absolute deviations (CLAD) estimator, and the Two-Part Model (2 PM) which especially take into account censoring issues (e.g. a high proportion of respondents report full health with a utility of 1) [28]. These three estimators are not used in this analysis because sample censoring is not an issue when AQoL-8D utility was scored in this study.

Model performance was assessed using the internal data, due to the lack of availability of an external validation dataset. The full sample was divided equally into five groups using computer-generated random numbers. In each group, $80 \%$ of the sample was assigned to the "estimation sample" that was used to generate the mapping algorithm, while the remaining $20 \%$ of the sample (assigned to the "validation sample") was used to predict AQoL-8D utilities based on the above algorithm. This procedure was repeated five times, so that each of the five random groups was used in the estimation and validation exercises.

Goodness-of-fit was examined by the mean absolute error (MAE) and the root mean square error (RMSE). 
The best fitting model was identified as the combination with the lowest combination of MAE and RMSE values. When there was a disagreement between the optimal chosen combination, the procedure used by Kay [29] was adopted, which used the RMSE of the validation analysis as the key criteria to measure model performance. All analyses were estimated in STATA version 12.1 (StataCorp LP, College Station, Texas, USA).

\section{Results}

\section{Patient characteristics}

A total of 254 subjects were recruited through the Australian centres. Of these, $177 \mathrm{iOAB}$ subjects with UI completed the I-QOL and AQoL instruments and were included in the present study. As shown in Table 1, the mean age of patients was 56 (SD: 14), with females comprising $85 \%$ of the samples. Participants experienced an average of 2.5 incontinence episodes per day (range 1 to $15, \mathrm{SD} 2$ ) and 1.9 nocturia episodes per night. The respondents had a mean I-QOL total score of 77.99 (SD 18.34 , range 23.86 to 100 ), and a mean AQoL-8D utility of 0.62 (SD 0.21, range 0.13 to 0.99 ), which is comparable to utility estimates previously reported in incontinent patients in Australia [8].

The distribution of the I-QOL total score and AQoL$8 \mathrm{D}$ utility of the study respondents is shown in Figure 1. The correlations between I-QOL scores and AQoL dimension values and utility are presented in Table 2 . Notably, all I-QOL subscales are significantly correlated with AQoL-8D dimensions $(\mathrm{p}<0.0001)$. The correlation ranges from 0.22 (between Avoidance and Limiting Behaviour in I-QOL and Mental Health in AQoL-8D) to 0.47 (between Avoidance and Limiting Behaviour in I-QOL and Independent Living in AQoL-8D). Overall, the I-QOL total score and AQoL-8D utility show moderately correlation with a coefficient of 0.50 .

\section{Mapping results}

In all of the model specifications the squared term of the I-QOL score and the age and gender variables were statistically insignificant and are not included in the results reported below. The goodness-of-fit statistics for the remaining models and statistical methods are reported in Table 3. The first three columns show the predicted mean minimum, maximum AQoL-8D utilities. With the exception of the MM-estimator, the predicted mean utilities, based on OLS estimators and GLM estimators, are either identical or very close to the observed (sample) mean utility. However, it should be noted that all predicted utilities tend to over predict the lowest, and under predict the highest boundary of the observed utility. This is not uncommon in the transformation analysis [30-32].

The MAE and RMSE are reported in the last two columns of Table 3. The MAE ranged from 0.1461 to
Table 1 General characteristics of patients

\begin{tabular}{|c|c|}
\hline Patient characteristic & $\mathrm{N}=177$ \\
\hline Mean age, years & 56.5 (SD: 13.7) \\
\hline Females & $85.3 \%$ \\
\hline Caucasian & $95.7 \%$ \\
\hline \multicolumn{2}{|l|}{ Mean years since diagnosis of $\mathrm{iOAB}, \mathrm{n}(\%)$} \\
\hline$\cdot<1$ year & $28(16)$ \\
\hline$\cdot 1-4$ years & $105(59)$ \\
\hline . 5-10 years & $28(16)$ \\
\hline$\cdot>10$ years & $16(9)$ \\
\hline \multicolumn{2}{|l|}{ History of anti-cholinergic medication use, n (\%) } \\
\hline - Used previously & $157(89)$ \\
\hline - Failed & $71(40)$ \\
\hline \multicolumn{2}{|l|}{ Education, n (\%) } \\
\hline - Elementary/High School & $129(73)$ \\
\hline - College/University degree & $35(20)$ \\
\hline - Post-graduate degree & $13(7)$ \\
\hline \multicolumn{2}{|l|}{ Income, n (\%) } \\
\hline$\cdot \leq \$ 50,000$ & $91(51)$ \\
\hline$\cdot \$ 50,001$ to $\$ 100,000$ & $35(20)$ \\
\hline$\cdot>\$ 100,000$ & $24(14)$ \\
\hline - Did not disclose & $27(15)$ \\
\hline Urinary incontinence, mean episodes per day (SD) & $2.5(2.2)$ \\
\hline Micturitions, mean episodes per day (SD) & $8.5(4.2)$ \\
\hline Nocturia ${ }^{a}, \mathrm{n}(\%)$ & $95(53.7)$ \\
\hline Urgency, mean episodes per day (SD) & $3.2(3.4)$ \\
\hline I-QOL score, mean (SD) & $78.0(18.3)$ \\
\hline AQoL-8D utility, mean (SD) & $0.62(0.21)$ \\
\hline
\end{tabular}

0.1479, whilst the RMSE varied from 0.1795 to 0.1831 . Although not reported in Table 2, the R-squared statistics for both models estimated using OLS were 0.2493 (Model 1) and 0.2509 (Model 2), which are within the acceptable range for mapping algorithms [11]. The MAE and RMSE were very similar across the various combinations of models and methods, with Model 2 (using IQOL subscale scores as key explanatory variables) based on GLM estimator having the lowest MAE (0.1461) and lowest RMSE (0.1795).

The goodness-of-fit results from the validation analysis are reported in Table 4. As shown in columns 4 and 5, the minimum MAE was obtained using the OLS estimate with Model 1, while the best fit using the RMSE criterion was achieved with the GLM estimate on Model 1. The relative small sample size, and the small variance in MAE/RMSE between models may have led to the differing conclusions between the full sample and validation analysis. As discussed in the Methods section, when there is a disagreement, the final decision was made based on 


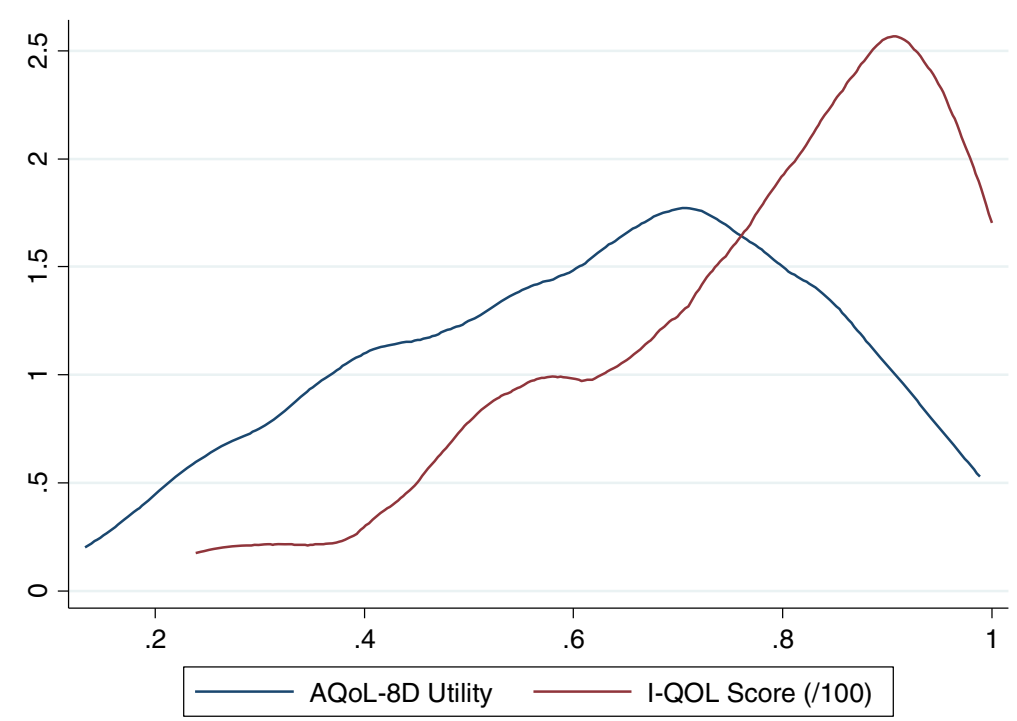

Figure 1 Kernal density of AQoL-8D utility and I-QOL total score.

the RMSE in the validation analysis. Thus, it is recommended that the mapping algorithm, based on I-QOL total score using GLM estimator (with Gaussian family and $\log$ link), should be adopted. From Table 4 this is given in the following equation:

AQoL-8D utility $=\exp (-1.28666+(1.011072 *$ IQOL/100 $))$.

The mapping equations corresponding to the goodnessof-fit results (shown in Table 3) are reported in Table 5. The scattergram correlation between the original AQoL$8 \mathrm{D}$ utility and the utility predicted by the preferred model is shown in Figure 2. The STATA commands for using the algorithm is given in Additional file 1.

\section{Discussion and conclusion}

With the increasing importance of health technology assessment in health system reforms and decision-making, the measurement of HRQOL are increasingly commonplace in clinical trials and public health intervention programs. However, respondent burden limits the number and type of QOL instruments included in clinical studies. Mapping analysis provides a method for transforming condition-specific quality of life scores into utilities

Table 2 Correlations between I-QOL and AQoL-8D

\begin{tabular}{|c|c|c|c|c|}
\hline & \multicolumn{3}{|l|}{ I-QOL subscale scores } & \multirow{2}{*}{$\begin{array}{l}\mathrm{I}-\mathrm{QOL} \\
\text { total score }\end{array}$} \\
\hline & Avoidance \& limiting behaviour & Psychosocial impacts & Social embarrassment & \\
\hline \multicolumn{5}{|c|}{ AQoL-8D dimensions } \\
\hline Independent Living & 0.4698 & 0.4584 & 0.4186 & 0.4876 \\
\hline Relationships & 0.3104 & 0.3377 & 0.2914 & 0.3406 \\
\hline Mental health & 0.2222 & 0.2805 & 0.3256 & 0.2944 \\
\hline Coping & 0.3082 & 0.3555 & 0.3971 & 0.3786 \\
\hline Pain & 0.4289 & 0.3878 & 0.3627 & 0.4269 \\
\hline Senses & 0.3371 & 0.3351 & 0.3808 & 0.3764 \\
\hline Happiness & 0.3103 & 0.3763 & 0.3734 & 0.3806 \\
\hline Self-Worth & 0.3597 & 0.4526 & 0.4468 & 0.4520 \\
\hline \multicolumn{5}{|c|}{ AQoL-8D super-dimensions } \\
\hline Physical & 0.4964 & 0.4614 & 0.4523 & 0.5089 \\
\hline Mental & 0.3227 & 0.3779 & 0.3716 & 0.3854 \\
\hline AQoL-8D utility & 0.4384 & 0.4813 & 0.4668 & 0.4993 \\
\hline
\end{tabular}

All correlation are significant $(p<0.0001)$. 
Table 3 Goodness-of-fit results for mapping from I-QOL score to AQoL-8D utility score

\begin{tabular}{|c|c|c|c|c|c|}
\hline & Mean AQoL-8D (1) & Min AQoL-8D (2) & Max AQoL-8D (3) & MAE (4) & RMSE (5) \\
\hline Observed & 0.6176 & 0.1331 & 0.9888 & - & - \\
\hline \multicolumn{6}{|c|}{ Method 1: OLS } \\
\hline Model 1 & 0.6176 & 0.3092 & 0.7430 & 0.1463 & 0.1808 \\
\hline Model 2 & 0.6176 & 0.2854 & 0.7243 & 0.1472 & 0.1806 \\
\hline \multicolumn{6}{|c|}{ Method 2: MM-estimator } \\
\hline Model 1 & 0.6185 & 0.2844 & 0.7543 & 0.1465 & 0.1810 \\
\hline Model 2 & 0.6231 & 0.2644 & 0.7079 & 0.1479 & 0.1831 \\
\hline \multicolumn{6}{|c|}{ Method 3: GLM ${ }^{+}$} \\
\hline Model 1 & 0.6176 & 0.3516 & 0.7591 & 0.1463 & 0.1804 \\
\hline Model 2 & 0.6174 & 0.3254 & 0.7387 & 0.1461 & 0.1795 \\
\hline
\end{tabular}

MAE: mean absolute error; RMSE: root mean squared error.

${ }^{\dagger}$ The Gaussian family with log link was used.

which can be used in Cost Utility Analysis. The present study provides a mapping algorithm to transform the I-QOL total score, commonly used in incontinence clinical trials $[33,34]$, to the AQoL-8D utility to enable the estimation of health state utility values.

In the present study, several model specifications and statistical methods were assessed. The best model result was obtained using the I-QOL total score as the key explanatory variable (Model 1), with the algorithm estimated with a robust GLM estimator (with Gaussian family and $\log$ link). As shown in Table 4, the model using the three I-QOL subscales also showed significant predictive ability. In particular, the Psychosocial Impact and Social Embarrassment subscales both showed significant associations, with the Psychosocial Impact subscale showing the strongest correlation with AQoL-8D $(r=0.48)$. This confirms the appropriateness of using the AQoL-8D to measure utility, as psycho-social QOL has been shown to be of importance for patients with UI and previous studies have shown AQoL-8D to be the most sensitive MAU instrument to psychosocial health among six generic preference-based instruments [22].

To date only one published study has mapped the I-QOL to preference-based HRQOL instruments (EQ-5D) [29]. The preferred model in the present study displays greater precision with both lower MAE and RMSE values ( 0.15 and 0.18 , respectively vs. 0.17 and 0.22 in Kay et al.). The study by Kay [29] adopted the 2 PM statistical method, due to the ceiling effects with the use of EQ-5D where $46 \%$ of subjects reported full health. As discussed in the Methods section, 2 PM was not considered in this study as no patient in this sample had full health on the AQoL-8D scale. In turn, the larger MAE and RMSE values in the study by Kay [29] are probably attributable to the methodological disadvantage of coping with the presence of significant ceiling effects. Consistent with the results in Kay [29] age and non-linear terms of I-QOL scores were found to be insignificant $(p>0.05)$.

Table 4 Goodness-of-fit results from validation analysis

\begin{tabular}{|c|c|c|c|c|c|}
\hline & Mean AQoL-8D (1) & Min AQoL-8D (2) & Max AQoL-8D (3) & MAE (4) & RMSE (5) \\
\hline Observed & 0.6176 & 0.1331 & 0.9888 & - & - \\
\hline \multicolumn{6}{|c|}{ Method 1: OLS } \\
\hline Model 1 & 0.6172 & 0.2858 & 0.7574 & 0.1511 & 0.1852 \\
\hline Model 2 & 0.6186 & 0.2945 & 0.7448 & 0.1538 & 0.1882 \\
\hline \multicolumn{6}{|c|}{ Method 2: MM-estimator } \\
\hline Model 1 & 0.6182 & 0.2565 & 0.7695 & 0.1516 & 0.1862 \\
\hline Model 2 & 0.6233 & 0.2742 & 0.7290 & 0.1530 & 0.1880 \\
\hline \multicolumn{6}{|c|}{ Method 3: $\mathrm{GLM}^{+}$} \\
\hline Model 1 & 0.6168 & 0.3291 & 0.7747 & 0.1514 & 0.1848 \\
\hline Model 2 & 0.6180 & 0.3235 & 0.7617 & 0.1536 & 0.1877 \\
\hline
\end{tabular}

MAE: mean absolute error; RMSE: root mean squared error.

${ }^{\dagger}$ The Gaussian family with log link was used. 
Table 5 Mapping equations from I-QOL score to AQoL-8D utility

\begin{tabular}{|c|c|c|c|c|c|c|}
\hline & \multicolumn{2}{|l|}{ OLS } & \multicolumn{2}{|c|}{ MM-estimator } & \multicolumn{2}{|l|}{$\mathrm{GLM}^{\ddagger}$} \\
\hline & Coeff. & SE & Coeff. & SE & Coeff. & SE \\
\hline \multicolumn{7}{|c|}{ Model 1 (using I-QOL total score) } \\
\hline $\mathrm{I}_{\mathrm{QOOL}}{ }^{+}$ & 0.5697422 & $0.0747355^{* *}$ & 0.6172410 & $0.0730065^{* *}$ & 1.0110720 & $0.1441272^{* *}$ \\
\hline Constant & 0.1732625 & $0.0598689 * *$ & 0.1370898 & $0.0615673^{*}$ & -1.2866600 & $0.1225249 * *$ \\
\hline \multicolumn{7}{|c|}{ Model 2 (using I-QOL subscale scores) } \\
\hline I-QOL-PS ${ }^{\dagger}$ & 0.3469097 & $0.1252910^{* *}$ & 0.5913308 & $0.0627952^{* *}$ & 0.6703759 & $0.2472593^{* *}$ \\
\hline I-QOL-SE & 0.1985825 & $0.0938378^{*}$ & & & 0.3521859 & $0.1691879^{*}$ \\
\hline Constant & 0.1787669 & $0.0690521^{* *}$ & 0.1165455 & $0.0564778^{*}$ & -1.3254590 & $0.1526178^{* *}$ \\
\hline
\end{tabular}

SE - standard errors. ${ }^{*} \mathrm{p}<0.05,{ }^{* *} \mathrm{p}<0.01{ }^{\dagger}$ The I-QOL total/subscale scores included in the regression model were calculated as original scores divided by 100. FThe Gaussian family with log link was used.

I-QOL-PS: I-QOL Psychosocial Impacts; I-QOL-SE: I-QOL Social Embarrassment.

There are several limitations to this study. Firstly, the sample size is modest, although mapping studies with smaller sample sizes have been reported upon in the literature $[11,23]$. Nevertheless, it is desirable that further mapping studies are conducted using larger samples to test the reliability of the mapping algorithm reported here. Secondly, the model performance is validated with internal data. Since mapping is data dependent, the choice of response sample may influence the calibration of mapping algorithms. A cross-validation study should therefore be conducted when an external dataset is available. Thirdly, the predicted AQoL-8D utilities do not capture the full range of observed AQoL-8D utilities. The overprediction of the lowest utilities and the under-prediction of the highest utilities may result in an underestimation of the utility gain.
Despite these qualifications, the goodness-of-fit measures reported in this study are within the ranges of previously published studies [11]. It is recognized that transformations cannot create information about dimensions which are not included in the instrument. As such the transformation of the I-QOL is the second best alternative to the use of an appropriate multi attribute utility instrument that is able to adequately capture changes in aspects of HRQOL affected by UI.

Results reported here indicate that the I-QOL can be mapped to AQoL-8D utility with acceptable precision at the group level in patients with urinary incontinence due to $\mathrm{iOAB}$. While both aggregated and individual level predictions of AQoL-8D utilities can be incorporated within the CUA, it is recommended that the group level predicted utility is more suitable [11].

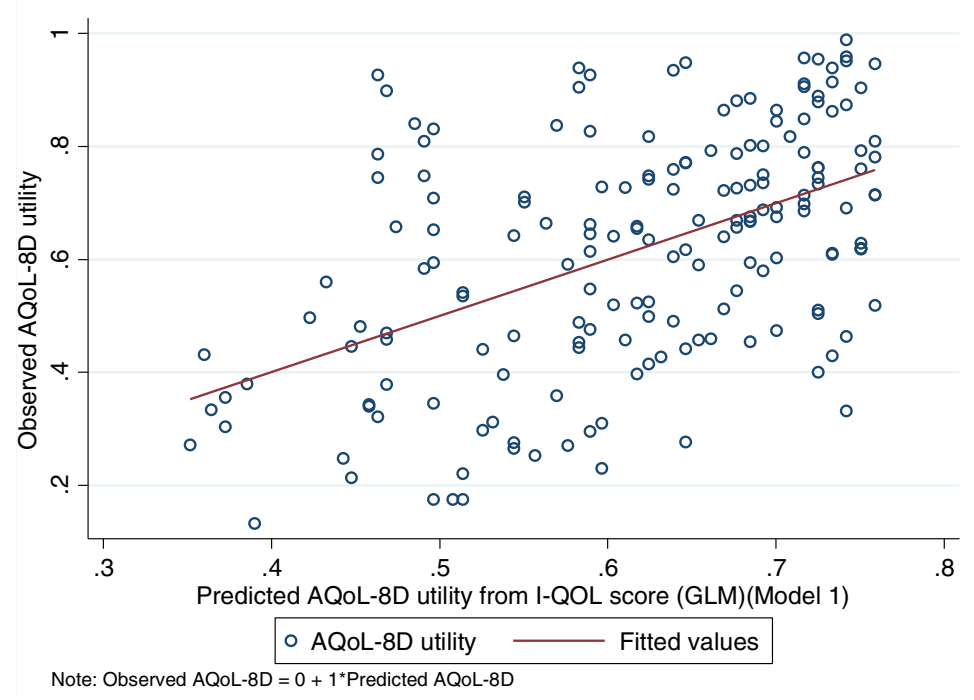

Figure 2 Scatter correlation between observed and predicted AQoL-8D utilities. 


\section{Additional file}

\section{Additional file 1: STATA algorithm.}

\section{Competing interests}

The current project was funded by Allergan. JT and KN are employees of Allergan. GC and JR are paid consultants to Allergan.

\section{Authors' contributions}

All authors have actively participated in the study and have made a substantial contribution to the study design and interpretation of results. GC and JT performed the statistical analyses. All authors were involved in the drafting or critical revision of the manuscript, and agreed to the final version.

\section{Author details}

${ }^{1}$ Flinders Health Economics Group, School of Medicine, Flinders University, Adelaide, Australia. ${ }^{2}$ Allergan Pty Ltd, Level 4, 810 Pacific Highway. Gordon, NSW 217 Melbourne, Australia. ${ }^{3}$ Allergan Pty Ltd, Pasir Panjang, Singapore.

${ }^{4}$ Centre for Health Economics, Monash University, Melbourne, Australia.

\section{Received: 9 May 2014 Accepted: 14 August 2014}

Published: 30 August 2014

\section{References}

1. Nitti W: The prevalence of urinary incontinence. Rev Urol 2001, 3(Suppl 1):S2.

2. Minassian VA, Drutz HP, Al-Badr A: Urinary incontinence as a worldwide problem. Int J Gynecol Obstet 2003, 82(3):327-338.

3. Aguzzi G, Simona B, Rosanna T: Systematic review of urinary incontinence and overactive bladder cost-of-illness studies. Open Pharmacoeconomics Health Econ J 2010, 2:11-24.

4. Hu T-W, Wagner TH, Bentkover JD, Leblanc K, Zhou SZ, Hunt T: Costs of urinary incontinence and overactive bladder in the United States: a comparative study. Urol 2004, 63(3):461-465.

5. Frick AC, Huang AJ, Van Den Eeden SK, Knight SK, Creasman JM, Yang J: Mixed urinary incontinence: greater impact on quality of life. J Urol 2009. 182(2):596.

6. Abrams P, Cardozo L, Wein A, Khoury S: Incontinence: 4th International Consultation on Incontinence. Paris, France: Health Publications; 2009.

7. Charalambous S, Trantafylidis A: Impact of urinary incontinence on quality of life. Pelviperineol 2009, 28(2):51-53.

8. Hawthorne G: Measuring Incontinence in Australia. Commonwealth of Australia; 2006

9. Stewart W, Van Rooyen J, Cundiff G, Abrams P, Herzog A, Corey R, Hunt T, Wein A: Prevalence and burden of overactive bladder in the United States. World J Urol 2003, 20(6):327-336.

10. Brazier J, Ratcliffe J, Salomon J, Tsuchiya A: Measuring and Valuing Health Benefits for Economic Evaluation. New York: Oxford University Press Inc; 2007.

11. Brazier JE, Yang Y, Tsuchiya A, Rowen DL: A review of studies mapping (or cross walking) non-preference based measures of health to generic preference-based measures. Eur J Health Econ 2010, 11(2):215-25.

12. Finkelstein MM, Skelly J, Kaczorowski J, Swanson G: Incontinence Quality of Life Instrument in a survey of primary care physicians. J Fam Pract 2002 51(11):952.

13. McCallum J, Millar L, Dong T, Burston L: National Continence Management Strategy 0 Independent Evaluation Advisor Final Report: Phases 1 and 2. Commonwealth of Australia; 2006.

14. Oh SJ, Ku JH: Is a generic quality of life instrument helpful for evaluating women with urinary incontinence? Qual Life Res 2006, 15(3):493-501.

15. Jimenez-Cidre M, Ng-Mak D, Sahai A, Degboe A, Smoth C, Tsai K, Herschorn S: Assessment of characteristics, treatment-seeking behavior, and healthcare utilization in an international cohort of subjects with overactive bladder. Curr Med Res Opin 2014. In press.

16. Wagner TH, Patrick DL, Bavendam TG, Martin ML, Buesching DE: Quality of life of persons with urinary incontinence: Development of a new measure. Urol 1996, 47(1):67-71.

17. Patrick DL, Martin ML, Bushnell DM, Yalcin I, Wagner TH, Buesching DP: Quality of life of women with urinary incontinence: further development of the incontinence quality of life instrument (I-QOL). Urol 1999, 53(1):71-76.
18. Bushnell D, Martin M, Summers K, Svihra J, Lionis C, Patrick D: Quality of Life of Women with Urinary Incontinence: Cross-cultural Performance of 15 Language Versions of the I-QOL. Qual Life Res 2005, 14(8):1901-1913.

19. Matza L, Brewster-Jordan J, Zyczynski T, Bavendam T: An updated review of quality-of-life questionnaires for urinary incontinence and overactive bladder: Which ones to use and why. Curr Bladder Dysfunct Rep 2006, 1(2):71-80

20. Richardson J, Sinha K, lezzi A, Khan MA: Modelling the utility of health states with the Assessment of Quality of Life (AQoL) 8D instrument: overview and utility scoring algorithm. In Research Paper 63, Center for Health Economics. Victoria, Australia: Monash University; 2011.

21. Richardson J, lezzi A, Khan MA, Maxwell A: Validity and reliability of the Assessment of Quality of Life (AQoL-8D) multi attribute utility instrument. Patient 2013, 7(1):85-96.

22. Richardson J, lezzi A, Khan MA, Maxwell A: Cross-national comparison of twelve quality of life instruments: MIC Paper 2 Australia. In Research Paper 78, Center for Health Economics. Victoria, Australia: Monash University; 2012.

23. Mortimer D, Segal L: Comparing the incomparable? A systematic review of competing techniques for converting descriptive measures of health status into QALY-weights. Med Decision Making 2008, 28(1):66-89.

24. Rabe-Hesketh S, Everitt B: A Handbook of Statistical Analyses Using Stata (Fourth Edition). Boca Raton, FL: Chapman \& Hall/CRC; 2007.

25. Yohai VJ: High breakdown-point and high efficiency robust estimates for regression. Ann Stat 1987, 15(2):642-56.

26. Chen G, Stevens K, Rowen D, Ratcliffe J: From KIDSCREEN-10 to CHU9D: creating a unique mapping algorithm for application in economic evaluation. Health Qual Life Out 2014, 12:134.

27. Fox J: Applied Regression Analysis and Generalized Linear Models (Second Edition). Thousand Oaks, CA: SAGE Publications; 2008.

28. Cameron AC, Trivedi PK: Microeconometrics: Methods and Applications. New York: Cambridge University Press; 2005.

29. Kay S, Tolley K, Colayco D, Khalaf K, Anderson P, Globe D: Mapping EQ-5D utility scores from the Incontinence Quality of Life Questionnaire among patients with neurogenic and idiopathic overactive bladder. Value Health 2013, 16(2):394-402.

30. Kaambwa B, Billingham L, Bryan S: Mapping utility scores from the Barthel index. Eur J Health Econ 2013, 14(2):231-41.

31. Starkie HJ, Briggs AH, Chambers MG, Jones P: Predicting EQ-5D Values Using the SGRQ. Value Health 2011, 14(2):354-60.

32. Versteegh $M$, Rowen D, Brazier J, Stolk E: Mapping onto Eq-5 D for patients in poor health. Health Qual Life Out 2010, 8(1):141.

33. Chapple C, Sievert KD, MacDiarmid S, Khullar V, Radziszewski P, Nardo C, Thompson C, Zhou J, Haag-Molkenteller C: OnabotulinumtoxinA $100 \mathrm{U}$ significantly improves all idiopathic overactive bladder symptoms and quality of life in patients with overactive bladder and urinary incontinence: a randomised, double-blind, placebo-controlled trial. Eur Urol 2013, 64(2):249-56.

34. Nitti WW, Dmochowski R, Herschorn S, Sand P, Thompson C, Nardo C, Yan X Haag-Molkenteller C, EMBARK Study Group: OnabotulinumtoxinA for the treatment of patients with overactive bladder and urinary incontinence: results of a phase 3, randomized, placebo controlled trial. J Urol 2013 89(6):2186-93

\section{doi:10.1186/s12955-014-0133-0}

Cite this article as: Chen et al:: Mapping of Incontinence Quality of Life (I-QOL) scores to Assessment of Quality of Life 8D (AQoL-8D) utilities in patients with idiopathic overactive bladder. Health and Quality of Life Outcomes 2014 12:133. 